\title{
La atención del alumnado inmigrante en Andalucía: medidas, actuaciones y grado de satisfacción
}

\author{
Dimitrinka Níkleva. Universidad de Granada \\ Sonia Peña García. Pontificia Universidad Católica del Ecuador
}

Recepción: 16.07.2018| Aceptado: 17.12.2018

Correspondencia a través de ORCID: Dimitrinka Níkleva

(iD 0000-0001-5458-7589

Citar: Níkleva, D. y Peña García, S. (2018). La atención del alumnado inmigrante en Andalucía: medidas, actuaciones y grado de satisfacción. ReiDoCrea, 8, 412-423.

\begin{abstract}
Resumen: En el ámbito educativo español, la presencia considerable de alumnado extranjero en todas las etapas educativas y en todas las comunidades autónomas ha originado una serie de medidas para afrontar toda la complejidad de esta realidad y atender al alumnado inmigrante. Según la normativa vigente, todas estas medidas se sitúan en el marco de la educación inclusiva. En este estudio se pretende dar a conocer el funcionamiento y las características de las Aulas Temporales de Adaptación Lingüística (ATAL) nombre que recibe las aulas de apoyo lingüístico en Andalucía. Los instrumentos de la investigación son varios cuestionarios que miden la opinión de: alumnos inmigrantes, sus familias, profesores en estas aulas y otros docentes de varios ámbitos educativos. Los resultados señalan los aspectos positivos y negativos de estas aulas, según los alumnos, sus familias y los profesores. Esto permite valorar la situación actual, detectar los aspectos mejorables y diseñar propuestas de mejora. Para enriquecer los datos del estudio se aporta algunos de los resultados del proyecto $I+D$ (referencia EDU2013-43868-P), titulado "La formación de los docentes de español para inmigrantes en diferentes contextos educativos".
\end{abstract}

Palabras clave: Educación cultural | Alumnado inmigrante

\section{Attention to immigrant students in Andalusia: procedures, actions and degree of satisfaction}

Abstract: In the Spanish educational field, the considerable presence of foreign students in all stages of the educational system in all of our autonomous regions has given rise to a number of measures to address the complexity of this reality and properly attend to immigrant students. According to current regulations, all these measures exist within the framework of 'inclusive education'. The aim of this study is to show the inner workings and characteristics of the linguistic support classrooms for immigrant students in Andalusia Spain, called 'Temporary classrooms for linguistic adaptation' (Aulas Temporales de Adaptación Lingüística (ATAL)). The research instruments were several questionnaires to measure the opinions of immigrant students, their families, teachers in these classrooms, and other teachers and lecturers from several educational fields. The results of this research show both the positive and negative aspects of these classrooms according to the students, their families, and the teachers. This allows the current situation to be assessed, aspects that need improvement to be determined and proposals to address them to be designed. To enhance the data of the study, some of the results of the R \& D project (reference EDU2013-43868-P), titled The training of teachers of Spanish for immigrants in different educational contexts, are provided.

Keywords: Cultural education | Immigrant students

\section{Introducción}

Las medidas diseñadas para la atención al alumnado inmigrante se pueden agrupar en tres ejes: la acogida, el aprendizaje del español como lengua vehicular y el mantenimiento de la cultura de origen. Este estudio se dedica al segundo eje, puesto que el apoyo lingüístico resulta fundamental para la competencia comunicativa y, de ahí, para la integración social del individuo. Este tipo de apoyo recibe distinta denominación en las diferentes comunidades autónomas, pero la esencia y los objetivos son muy parecidos: Aulas Temporales de Adaptación Lingüística (ATAL) en Andalucía y Extremadura; aulas de enlace en Madrid; Aulas Temporales de Inmersión Lingüística (ATIL) en Asturias; Aulas de Acogida en Cataluña, Islas Baleares y Murcia; Aulas de Inmersión Lingüística (AIL) en Navarra y La Rioja; Aulas de Adaptación 
Lingüística y Social (Aulas ALISO) en Castilla-León; Grupos de Adaptación de la Competencia Curricular y Grupos de Adquisición de las Lenguas en Galicia; Programas de Acogida al Sistema Educativo (PASE) en la Comunidad Valenciana y Programa de Refuerzo Lingüístico en el País Vasco. En este estudio optamos por describir el caso de la Comunidad Autónoma de Andalucía, es decir, de las Aulas Temporales de Adaptación Lingüística (ATAL). En el funcionamiento de estas aulas subyacen los fundamentos de la enseñanza de segundas lenguas y de la educación inclusiva. Contribuyen a la integración lingüística y social de los alumnos extranjeros y proporcionan un espacio de seguridad donde disminuye la ansiedad producida por las dificultades de comunicación.

\section{Las ATAL en la comunidad autónoma de Andalucía}

Las transformaciones sociales que se han venido produciendo desde hace años en Andalucía han contribuido a proponer un nuevo concepto de escuela: aquella que se basa en la calidad de la enseñanza y en la integración intercultural para un alumnado cada vez más heterogéneo (Cruz del Pino, 2011).

Según el artículo 3 de la Orden 15 de enero de 2007, que regula el funcionamiento de las ATAL, todos los centros que acogen alumnado inmigrante deberán planificar actuaciones específicas de acogida para facilitar la escolarización, integración y adaptación del alumnado. Los principales objetivos de dichas actuaciones serán:

1. Facilitar la escolarización del alumnado inmigrante en las mismas condiciones que el alumnado autóctono.

2. Favorecer la acogida, principalmente la integración social y escolar.

3. Favorecer la convivencia en el centro.

4. Fomentar la participación del alumnado en las actividades escolares y extraescolares.

5. Fomentar la implicación de las familias en el entorno escolar.

6. Potenciar las relaciones institucionales del centro con las autoridades municipales, servicios sociales, servicios de salud y otras instituciones que beneficien la inclusión escolar del alumnado inmigra.

\section{Normativa y funcionamiento de las ATAL}

Según lo establecido en la normativa vigente, en la Orden de 15 de enero de 2007, de la Consejería de Educación de la Junta de Andalucía este programa está dirigido:

A la enseñanza y aprendizaje del español como lengua vehicular, vinculados a profesorado específico, que permiten la integración del alumnado inmigrante en el centro su incorporación a los ritmos y actividades de aprendizaje propios del nivel en el que se encuentre escolarizados atendiendo a su edad y a su competencia curricular (Artículo 5, punto 1). Asimismo, establece que:

Estos programas deberán realizarse en el aula ordinaria. Se podrán organizar grupos de apoyo atendidos por profesorado específico para llevar a cabo la atención fuera del aula ordinaria cuando circunstancias especiales en la compresión-expresión de la lengua española así lo aconsejen (Artículo 5, punto 2).

Sin embargo, en la práctica, estas "circunstancias especiales" parecen darse en la mayoría de los casos, puesto que el apoyo que se ofrece al alumnado inmigrante se realiza fuera del aula ordinaria. Este modelo de atención externo es conocido como 
enfoque segregacionista (Arroyo,2010) frente a la modalidad inclusiva que sería la atención en el aula ordinaria.

Las aulas ATAL tienen actividad en los centros públicos andaluces que imparten enseñanzas tanto en educación primaria como en educación secundaria obligatoria (entre $3 .^{\circ}$ de Primaria y $4 .^{\circ}$ de ESO). Los centros que acogen alumnado inmigrante deberán desarrollar ciertas medidas y actuaciones para favorecer su acceso, permanencia y promoción en el sistema educativo. Con ello, pretenden fomentar el valor de la interculturalidad, el respeto a la diversidad cultural y la convivencia entre diferentes culturas. Cada una de estas actuaciones deberá reflejarse en todos y cada uno de los apartados del proyecto de centro, que habrá de incluir acciones que desarrollen, por lo menos, los siguientes aspectos: a) La acogida del alumnado inmigrante: de forma que facilite el proceso de escolarización e integración. b) El aprendizaje del español como lengua vehicular: los centros que acogen alumnado inmigrante deberán planificar actuaciones específicas. Estas actuaciones se podrán llevar a cabo mediante las aulas ATAL, actividades extraescolares o mediante la utilización de las TIC. c) El mantenimiento de la cultura de origen: los centros podrán desarrollar actuaciones específicas para potenciar el aprendizaje de la cultura de origen y difundir información de cada una de las culturas por toda la comunidad educativa.

Como se ha mencionado previamente, el programa de ATAL se deberá realizar en el aula ordinaria, pero se podrán organizar grupos de apoyo fuera de ella en casos excepcionales y para ello contarán con profesores específicos.

La asistencia a estos grupos de apoyo será de 10 horas semanales para la educación primaria y de 15 horas en educación secundaria obligatoria. El alumnado inmigrante solo podrá participar en el programa durante un curso, pero podrá permanecer hasta un máximo de dos cursos cuando concurran las siguientes circunstancias, siempre bajo la previa autorización del servicio de inspección:

1. Enfermedad que haya dado lugar a faltas continuadas a clase.

2. Absentismo escolar por distintos motivos.

3. Escasa o nula escolarización en su país de origen.

4. Inscripción en el centro a lo largo del segundo o tercer trimestre del curso anterior.

Con los resultados obtenidos en la evaluación continua se podrá determinar la finalización de la asistencia. Esta decisión será tomada por la jefatura de estudios, en colaboración con el profesorado de ATAL, el profesorado de las tutorías y del personal de orientación.

Las aulas temporales de adaptación lingüística se ofrecen a partir del segundo ciclo en educación primaria y hasta el final de la educación secundaria obligatoria. El proceso de selección del alumnado inmigrante se realizará a través del nivel de competencia lingüística y será llevado a cabo por el profesor encargado de la tutoría, en colaboración con profesionales de orientación de cada centro. El alumnado con nivel inferior a B1 tendrá preferencia para participar en las aulas ATAL, ya que el grupo de apoyo no excederá de los 12 alumnos.

El nivel de competencia lingüística será ponderado según las equivalencias de los niveles fijados en el Marco Común Europeo de Referencia para las Lenguas (MCER) 
(Consejo de Europa, 2002) pero para su utilización en las aulas ATAL, estos seis niveles han sido simplificados y reducidos a cuatro.

\begin{tabular}{|ll|}
\hline \multicolumn{2}{|l|}{ Cuadro 1.1. Correspondencia entre los niveles lingüísticos de ATAL y del MCER. } \\
\hline Niveles ATAL & Niveles MCER \\
\hline Nivel 0 & Ausencia de las características del nivel A1 \\
Nivel 1 & A1 \\
Nivel 2 & A2 \\
Nivel 3 & B1 \\
\hline
\end{tabular}

\section{Rol del docente}

De acuerdo con la Orden, el funcionamiento idóneo de las ATAL supone contar con profesores itinerantes destinados a esta función; pero nos surge la siguiente cuestión ¿A qué se refiere con profesor itinerante específico? Según la Orden el profesorado de ATAL no requiere ninguna cualificación o formación específica para impartir el español como segunda lengua y ni siquiera es obligatorio que sean profesores de Lengua española. A diferencia de otras comunidades autónomas que sí demanda, formación específica, como es el caso de Castilla la Mancha (formación en educación compensatoria) y Asturias (formación en atención a la diversidad), etc. (Arroyo, 2011). Por otro lado, cabe mencionar que la Comunidad de Madrid y el País Vasco son las únicas comunidades autónomas que sí exigen formación en español como lengua extranjera para impartir el español como segunda lengua en las aulas de acogida. (Arroyo González, 2011). Respecto a la formación del profesorado en estas aulas Arroyo (2011: 138) concluye:

"Escasa preparación del profesorado en esta materia. La normativa no hace referencia a ninguna formación específica previa, salvo excepciones (la comunidad de Madrid exige que el profesorado tenga formación en español como Lengua Extranjera). Estas funciones están siendo desarrolladas por profesorado de Educación Compensatoria, de Lengua Extranjera, de Atención a la Diversidad. Se debe incidir en la formación especifica del profesor de español como lengua Extranjera".

El profesorado de las aulas temporales de adaptación lingüística será el responsable de todas aquellas funciones relacionadas con el alumnado inmigrante con carencias en el conocimiento del español como lengua vehicular (Artículo 7, punto 1). A continuación, se presentan algunas de las tareas que habrá de desempeñar:

1. Enseñar la lengua española de tal manera que genere la correcta integración del alumnado inmigrante en el ámbito escolar y social.

2. Atender las dificultades de aprendizaje del alumnado inmigrante.

3. Elaborar las programaciones de las aulas ATAL adaptándolas a las necesidades del alumno. Dichas programaciones deberán ser incluidas en el plan de orientación y acción tutorial del centro.

4. Elaborar un informe de cada alumno, así como cumplimentar una carpeta de seguimiento que incluya asistencia y evolución.

5. Elaborar una memoria al finalizar el curso, la cual será incluida en la memoria final del centro.

A pesar de que el profesorado de ATAL es el responsable casi exclusivo de los procesos de inmersión lingüística y de la integración social del alumnado inmigrante con desconocimiento de la lengua, en muchas ocasiones no cuentan con un marco institucional adecuado al desarrollo de sus funciones, lo cual llega a dificultar su labor y con ello el logro de los objetivos del programa. 
La jefatura de estudios de cada centro junto con el asesoramiento del departamento de orientación en educación secundaria obligatoria o del orientador de referencia en educación primaria serán los encargados de la coordinación y actuaciones desarrolladas por los profesores de ATAL. El profesorado deberá asistir siempre que le sea posible a las reuniones de ciclo (en Primaria), de tutorías (en Secundaria) y a las sesiones de evaluación del alumnado atendido en el curso escolar. Al atender alumnos de varios centros habitualmente ubicados en una misma zona, el profesorado decidirá a qué centro asistirá para atender a claustros y evaluaciones. Por normativa el profesorado de ATAL no podrá realizar sustituciones del profesorado del centro, salvo con autorización expresa de La Delegación Provincial.

Las Delegaciones Provinciales de la Consejería de Educación serán los encargados de la organización del profesorado de ATAL y determinarán el número de horas por cada centro. El equipo técnico provincial de orientación educativa y profesional será el que informe del número de horas establecidas para la atención al alumnado inmigrante en cada centro, así como las horas determinadas a la coordinación, asesoramiento y confección de documentos, según lo establecido en la normativa vigente.

\section{Enfoque inclusivo frente al enfoque segregacionista}

Como se ha mencionado previamente en el apartado de la normativa y funcionamiento de las ATAL, en la práctica se aplican dos enfoques para atender al alumnado inmigrante, el enfoque inclusivo y el segregacionista.

El enfoque inclusivo, conocido como cooperativo, se ha recomendado para trabajar con el alumnado inmigrante dentro del aula ordinaria, sin embargo, el enfoque que predomina en los centros andaluces y en algunas comunidades -como es el caso de Madrid donde se ha adoptado de forma institucional (Arroyo,2010) es el enfoque segregacionista.

Existen estudios que han comparado los dos enfoques y muestran que el enfoque inclusivo aporta numerosas ventajas y un mejor rendimiento académico, no solo para el alumnado inmigrante extranjero, sino que también beneficia al alumnado autóctono (Barrios y Morales, 2012).

Otros estudios muestran el modelo segregacionista (atención fuera del aula ordinaria) como modelo homogeneizador y persistente en relación a la aceptación de la diversidad, pero a su vez promueve la no colaboración del resto de profesores en la adquisición de competencia lingüística (García y Moreno, 2003; García, Moreno y Sánchez, 2008).

Entre los estudios realizados sobre el mismo tema, nos parece no solo oportuno, sino necesario incluir el grado de satisfacción con estas aulas. Para ello ofrecemos a continuación los resultados de un estudio reciente del 2014/2015 que la Dirección General de Participación y Equidad solicita con el objetivo de conocer el grado de satisfacción del profesorado en las ATAL, del alumnado en estas aulas y de sus familias, con la finalidad de introducir las mejoras pertinentes (Agencia Andaluza de Evaluación Educativa, 2015).

\section{Objetivos}

Dar a conocer el funcionamiento y las características de las Aulas Temporales de Adaptación Lingüística (ATAL) nombre que recibe las aulas de apoyo lingüístico en Andalucía. 


\section{Métodos}

La muestra del alumnado es de el 95\% (sexo masculino: $53 \%$, sexo femenino: $47 \%$ ). Participan también las familias de la mayoría del alumnado (94\%).

En cuanto al profesorado, no se ha extraído una muestra, puesto que ha participado el profesorado en su totalidad de 211 profesores durante el curso 2014/2015.

\section{Resultados}

\section{Respuesta de los alumnos}

Ningún alumno considera mala la acogida del profesorado. El 82,45 \% la considera muy buena. El porcentaje baja en cuanto a la acogida de los compañeros: muy buena $(45,2 \%)$.

Las ATAL se regulan por la Orden de 15 de enero de 2007 y allí se establece que deberán realizarse en el aula ordinaria, aunque se permite organizar grupos de apoyo fuera del aula ordinaria cuando circunstancias especiales en la comprensión/expresión de la lengua española así lo aconsejen. Con el objetivo de estudiar qué enfoque inclusivo o segregacionista- predomina en la práctica se hizo la siguiente pregunta: ¿Asistes al aula de español (ATAL) separado de tu grupo?

Las opciones de respuesta fueron "Si" y "No". Un 9,1\% del alumnado encuestado recibe la atención en el aula ordinaria y un $90,9 \%$ lo hace en grupos de apoyo fuera del aula ordinaria. Este resultado revela que en la práctica predomina el enfoque segregacionista, el no recomendado por la Orden. Pero es muy importante el dato que aportan los propios alumnos: un $76 \%$ de este alumnado no está de acuerdo o está poco de acuerdo con que se realice la atención en el aula ordinaria.

Es llamativo el parecido en la respuesta de "estoy aprendiendo o he aprendido el español que necesito". Es ligeramente mayor en los alumnos atendidos en el aula ordinaria $(68,3 \%)$ frente a los alumnos atendidos fuera del aula ordinaria $(62,8 \%)$.

La tabla 1 recoge los resultados de la comparación de los dos enfoques.

\begin{tabular}{|l|c|c|}
\hline Tabla 1 Enfoque inclusivo vs. enfoque segregacionista. \\
\hline Pregunta & $\begin{array}{c}\text { En el aula ordinaria } \\
\text { (enfoque inclusivo) }\end{array}$ & $\begin{array}{c}\text { Fuera del aula ordinaria } \\
\text { (enfoque segregacionista) } \\
\text { Aula ATAL }\end{array}$ \\
\hline Estoy aprendiendo o he aprendido el español que necesito. & $68,3 \%$ & $62,8 \%$ \\
\hline $\begin{array}{l}\text { Se realizan actividades suficientes para el conocimiento y } \\
\text { valoración de las diferentes culturas. }\end{array}$ & $63,4 \%$ & $38,4 \%$ \\
\hline $\begin{array}{l}\text { Mi profesor o profesora de español (ATAL) me ayuda a } \\
\text { comunicarme con los otros profesores. }\end{array}$ & $66,6 \%$ & $60,0 \%$ \\
\hline $\begin{array}{l}\text { En el aula se realizan actividades para } \\
\text { mantener la cultura de tu país. }\end{array}$ & $51,2 \%$ & 37,95 \\
\hline Me siento bien atendido en el aula. & $81,0 \%$ & $81,8 \%$ \\
\hline
\end{tabular}

Hemos de destacar también los siguientes resultados: casi el $67 \%$ del alumnado de la muestra que es atendido fuera del aula ordinaria manifiesta que no se siente perdido cuando no está en el aula de ATAL; el $85 \%$ aproximadamente de este alumnado afirma que no se siente extraño o apartado cuando sale del aula ordinaria para acudir al aula de español de ATAL. 
Otros datos positivos que hemos de mencionar tienen que ver con el rendimiento académico en otras asignaturas. Más del $95 \%$ del alumnado de la muestra que es atendido fuera del aula ordinaria afirma que la asistencia al aula de ATAL le ha servido de ayuda para progresar en otras asignaturas.

Más de las tres cuartas partes de este alumnado utiliza materiales preparados por el profesorado de ATAL en las asignaturas impartidas en el aula ordinaria. Es un dato que confirma la implicación y el buen hacer de este profesorado.

Las preferencias de los alumnos apuntan a que son necesarios dos profesores a la vez $(61 \%)$, pero que "sería mejor enseñar español aparte".

\section{Respuestas de las familias}

La mayoría de las respuestas manifiestan una gran satisfacción con la labor del profesorado respecto a la acogida y la integración de sus hijos.

Respecto al comienzo en el programa ATAL, más del $68 \%$ de las familias encuestadas opina que debería ser antes de $3^{\circ}$ de Primaria.

Más del $88 \%$ de las familias entrevistadas considera que sus hijos están aprendiendo o han aprendido el español que necesitan y solo un $2 \%$ considera que no.

El $26,8 \%$ opina que se realizan suficientes reuniones con las familias ("muy de acuerdo"). El 40,6\% está de acuerdo. Un 11\% de las familias considera que las reuniones no son suficientes.

Más del $70 \%$ de las familias afirma que su hijo se relaciona con los compañeros de clase fuera del colegio o instituto. Esta misma afirmación fue contestada por el $64 \%$, del alumnado.

Más del $59 \%$ de las familias encuestadas se relaciona con las familias de otros alumnos y alumnos extranjeros, mientras que un $41 \%$ lo hace en menor medida.

En la tabla 2 recogemos los resultados más relevantes en la comparación de los dos enfoques, tal como se ha hecho anteriormente para los alumnos. Se proporciona solo el porcentaje que corresponde a "muy de acuerdo" sumado con el "de acuerdo".

\begin{tabular}{|lll|}
\hline Tabla 2 Aula ATAL y aula ordinaria & & \\
\hline Pregunta & $\begin{array}{l}\text { En el aula ordinaria } \\
\text { (enfoque inclusivo) }\end{array}$ & $\begin{array}{l}\text { Fuera del aula ordinaria } \\
\text { (enfoque segregacionista) } \\
\text { Aula ATAL }\end{array}$ \\
\hline $\begin{array}{l}\text { El trabajo desarrollado en el aula se ajusta a lo que } \\
\text { necesita su hija o hijo }\end{array}$ & $56,1 \%$ & $93,5 \%$ \\
$\begin{array}{l}\text { Su hijo está bien atendido } \\
\begin{array}{l}\text { En general, lo conseguido en el aula es suficiente para } \\
\text { continuar los estudios con normalidad }\end{array}\end{array}$ & $97,6 \%$ & $96,7 \%$ \\
\hline
\end{tabular}

Otras opiniones de las familias que hemos de destacar serían las siguientes:

- Estar en el aula de español (ATAL) le ha servido de ayuda a su hijo (97,2 \% "muy de acuerdo" y "de acuerdo"). 
- Que el profesor de español (ATAL) esté en el aula junto con el profesor de la materia le ha servido de ayuda a su hija o hijo (92,3 \% "muy de acuerdo" y "de acuerdo").

Respecto a algunas preguntas que se han realizado solo a familias, cuyos hijos son atendidos fuera del aula ordinaria, destacamos los siguientes resultados del estudio de la Agencia Andaluza de Evaluación Educativa (2015): Más del $81 \%$ opina que la duración máxima del programa (tiempo de estancia total en el aula de ATAL) es adecuada. Cerca del $78 \%$ cree que su hijo no se siente extraño o apartado cuando sale de su aula ordinaria para acudir al aula de español.

Esta misma cuestión fue planteada en el cuestionario dirigido al alumnado y, tal como se ha dicho anteriormente, este porcentaje fue del $85 \%$. Un $58 \%$ no está de acuerdo o está poco de acuerdo con que sus hijos sean atendidos solo en el aula ordinaria frente a un $42 \%$ de las familias que considera que el alumnado que va al aula de español (ATAL) debería ser atendido dentro del aula ordinaria, con todos los demás.

En el citado estudio de la Agencia Andaluza de Evaluación Educativa (2015) se han formulado también preguntas solo a las familias del alumnado atendido dentro del aula ordinaria. Los datos indican los siguientes resultados más relevantes: El 77,3\% de las familias cree que es necesario que haya dos profesores en el aula, y que uno de ellos sea de español. El $62,8 \%$ de estas familias opina que sería mejor formar un grupo de alumnos que no saben español y enseñarles aparte y que después se incorporen al aula con todos los demás ("muy de acuerdo" y "de acuerdo").

\section{Respuesta del profesorado de ATAL}

El profesorado ATAL ha participado en su totalidad (97\%). Predominan las mujeres $(81 \%)$ frente a los hombres (19\%).

Respecto al comienzo del programa que actualmente es en $3 .^{\circ}$ de Educación Primaria, el $56,4 \%$ opina que debería ser en $1 .^{\circ}$ de Primaria y casi un $18 \%$ considera que en Educación Infantil. Recordemos que en el cuestionario para las familias se hizo esta misma pregunta y más del $68 \%$ opinó que el comienzo en ATAL debería ser antes de $3 .^{\circ}$ de Primaria. Aquí se observa que casi el $88 \%$ del profesorado considera lo mismo.

Sobre la colaboración de las familias en la educación de sus hijos, más del $9 \%$ del profesorado está "muy de acuerdo" y cerca del 53 \%, "de acuerdo". Es relevante el dato que señala que más de la tercera parte del profesorado de ATAL considera mejorable la colaboración de las familias.

El profesorado en su totalidad considera que el ATAL ha favorecido la integración escolar de los alumnos y más del $90 \%$ opina que contribuye al mantenimiento de su cultura de origen.

Casi las tres cuartas partes del profesorado de ATAL consideran que la duración máxima del programa (tiempo de estancia total del alumnado en el aula de ATAL) no es la adecuada y cerca del $72 \%$ cree que el número de horas semanales en el aula de ATAL es insuficiente frente a solo un $28 \%$ del profesorado de ATAL y un $32 \%$ de los tutores y tutoras que sí consideran adecuado el número de horas.

Otro resultado por destacar es que más del $66 \%$ del profesorado considera que lo conseguido tras la finalización del Programa ATAL, o sea, la competencia adquirida en 
español como lengua vehicular, no es suficiente para que el alumnado extranjero tenga acceso al currículo establecido.

Hemos de destacar también los resultados de dos preguntas sobre la preparación del profesorado y su formación (inicial y permanente): A la pregunta "En general, el profesorado de ATAL tiene la preparación suficiente para atender al alumnado extranjero" un $87,6 \%$ del profesorado considera que "sí" frente a un $12,4 \%$ que opina que "no".

La siguiente pregunta "El profesorado ATAL recibe suficiente formación (inicial y permanente" fue contestada con un "sí" por el $52,5 \%$ y con un "no" por un número considerable de profesores $(47,5 \%)$.

Estas respuestas contradicen los resultados del proyecto I+D en el que se enmarca nuestro estudio donde en general se confirma que la preparación inicial es insuficiente (incluida la titulación universitaria que no ofrece formación al respecto), los requisitos para optar al puesto de ATAL no contemplan una formación específica en la enseñanza de segundas lenguas y la formación continua se centra más en temas de TIC, interculturalidad, etc., pero sigue sin atender la necesidad de formación lingüística y didáctica específica en el ámbito del español como segunda lengua.

Siguiendo con el estudio de Agencia Andaluza de Evaluación Educativa (2015), se observa también que cerca del $93 \%$ del profesorado considera que existen suficientes recursos humanos en los centros para atender las necesidades del alumnado extranjero y que un $77 \%$ cuenta con materiales suficientes.

La opinión del profesorado de ATAL sobre si el alumnado debería ser atendido dentro del aula ordinaria (con dos profesores) se distribuye de la siguiente manera: "sí" $(49,1$ $\%)$ y "no" (50,9 \%). Aun así, el $89 \%$ de los que están a favor del aula ordinaria considera que el alumnado de nivel 0 debe permanecer en el aula de ATAL, excepto en las materias que favorecen las relaciones personales.

En la tabla 3 señalamos algunas ventajas indicadas por el profesorado a favor del ATAL y a favor del aula ordinaria (datos extraídos del informe de la (Agencia Andaluza de Evaluación Educativa, 2015).

\begin{tabular}{|ll|}
\hline \multicolumn{2}{|l|}{ Tabla 3 Ventajas según el profesorado a favor del ATAL y a favor del aula ordinaria } \\
\hline Profesorado a favor del ATAL & Profesorado a favor del aula ordinaria \\
\hline El esfuerzo en programar la enseñanza en doble & El esfuerzo en programar esta modalidad de \\
docencia no se ve recompensado por los resultados que enseñanza se ve recompensado por los resultados & en \\
se obtienen (66 \%) & que se obtienen $(98 \%)$ \\
El aprendizaje del español es más efectivo y el trato del & El apoyo dentro del aula ordinaria, las relaciones y la \\
alumnado es más personal y diferenciado en el aula de & $\begin{array}{l}\text { integración de todo el alumnado mejoran } \\
\text { ATAL }(100 \%)\end{array}$ \\
\hline
\end{tabular}

\section{Resultados del Proyecto I+D}

Para enriquecer los datos del estudio anterior de la Consejería de Educación de la Junta de Andalucía proporcionamos a continuación solo algunos de los resultados del I+D (referencia EDU2013-43868-P), titulado La formación de los docentes de español para inmigrantes en diferentes contextos educativos.

Hay que tener en cuenta que los participantes en este estudio son profesores de español en distintos contextos educativos (no solo ATAL) (tabla 4). El cuestionario fue 
rellenado en 2015 por 131 profesores, de los cuales 5 son ATAL. Algunos de ellos no son profesores en activo, pero son licenciados en Filología española o Graduados en el Grado de Educación Primaria y muchos han realizado un máster de español como lengua extranjera.

Presentamos datos del análisis descriptivo, ordenados de mayor a menor frecuencia.

\begin{tabular}{|lcc|}
\hline \multicolumn{3}{|c|}{ Tabla 4 Actualmente trabaja como profesor de español } \\
\hline Variable & Frecuencia & $\%$ \\
\hline No trabaja (pero docente de titulación) & 35 & 26,72 \\
Universidad & 19 & 14,5 \\
Educación Secundaria & 17 & 12,98 \\
Educación Primaria & 12 & 9,16 \\
Escuela Oficial de Idiomas & 11 & 8,4 \\
Centro de Lenguas Modernas & 10 & 7,63 \\
Academia ELE & 8 & 6,11 \\
ONG & 9 & 6,87 \\
ATAL o aula de enlace & 5 & 3,82 \\
Varias categorías & 5 & 3,82 \\
\hline
\end{tabular}

Queremos hacer hincapié en los siguientes resultados sobre la preparación y la formación continua que recibe el profesorado y que no coinciden con el estudio citado anteriormente de la Agencia Andaluza de Evaluación Educativa (2015), donde el $87,6 \%$ del profesorado considera que tiene preparación suficiente y el $52,5 \%$ manifiesta tener suficiente formación inicial y permanente.

Con nuestro proyecto hemos obtenido los siguientes resultados: El 65,31\% está "muy en desacuerdo" con la formación general recibida durante la carrera universitaria para atender a alumnos inmigrantes (tabla 5).

\begin{tabular}{|lcc|}
\hline $\begin{array}{l}\text { Tabla 5. Pregunta: Durante su carrera universitaria recibió formación general para atender a alumnos } \\
\text { inmigrantes }\end{array}$ & \multicolumn{1}{c|}{} \\
\hline Variable & Frecuencia & 65,31 \\
\hline Muy en desacuerdo & 96 & 11,56 \\
Algo en desacuerdo & 17 & 10,2 \\
Algo de acuerdo & 15 & 7,48 \\
Ni acuerdo ni desacuerdo & 11 & 5,44 \\
Muy de acuerdo & 8 & \\
\hline
\end{tabular}

Solo el $28 \%$ se considera preparado para ser profesor de español para inmigrantes ("muy de acuerdo") (tabla 6).

\begin{tabular}{|lcc|}
\hline \multicolumn{3}{|c|}{ Tabla 6. Tiene la formación necesaria y suficiente para ser profesor de español para inmigrantes } \\
\hline Variable & Frecuencia & $\%$ \\
Algo de acuerdo & 45 & 29,8 \\
Ni acuerdo ni desacuerdo & 36 & 23,84 \\
Muy de acuerdo & 28 & 18,54 \\
Algo en desacuerdo & 25 & 16,56 \\
Muy en desacuerdo & 17 & 11,26 \\
\hline
\end{tabular}

Respecto al número aproximado de horas de formación sobre la enseñanza del español como lengua extranjera o como segunda lengua que han recibido, las respuestas se distribuyen de la siguiente manera (tabla 7 ):

Tabla 7. Horas de formación sobre la enseñanza del español como lengua extranjera o como segunda lengua 


\begin{tabular}{|lcc|}
\hline Variable & Frecuencia & $\%$ \\
\hline $101-500$ & 31 & 20,67 \\
$1-30$ & 23 & 15,33 \\
$501-1000$ & 23 & 15,33 \\
$>1000$ & 22 & 14,67 \\
0 & 22 & 14,67 \\
$31-60$ & 15 & 10 \\
$61-100$ & 14 & 9,33 \\
\hline
\end{tabular}

Los datos sobre el número de horas de formación continua apuntan lo siguiente (tabla 8):

\begin{tabular}{|lcc|}
\hline \multicolumn{3}{|l|}{ Tabla 8. Número aproximado de horas de formación continua del profesorado que ha recibido en cursos de } \\
los CEP/CPR (centros de profesores) & & \\
\hline Variable número de horas & Frecuencia (sujetos) & Porcentaje \\
0 & 77 & 53,85 \\
$101-500$ & 20 & 13,99 \\
$31-60$ & 14 & 9,79 \\
$1-30$ & 9 & 6,29 \\
$501-1000$ & 8 & 5,59 \\
$61-100$ & 7 & 4,9 \\
\hline
\end{tabular}

Para nuestro estudio es importante conocer la temática de los cursos de formación continua (tabla 9) y observamos que predominan las TIC.

\begin{tabular}{|lcc|}
\hline \multicolumn{3}{l}{ Tabla 9. ¿Cuál era la temática predominante en los cursos de formación continua que ha recibido en los } \\
CEPICPR, etc.? & Frecuencia & $\%$ \\
\hline Variable & 14 & 20,29 \\
TIC & 11 & 15,94 \\
Gramática & 10 & 14,9 \\
Otro & 9 & 13,04 \\
Metodología & 6 & 8,7 \\
Recursos y materiales & 4 & 5,8 \\
Destrezas & 4 & 5,8 \\
Interculturalidad o cultura & 3 & 4,35 \\
Cultura materna de los inmigranes & 2 & 2,9 \\
Dinámica de grupos & 1 & 1,45 \\
Competencias & 1 & 1,45 \\
Inclusión & 1 & 1,45 \\
Investigación & 1 & 1,45 \\
Legislación & 1 & 1,45 \\
Programación & 1 & 1,45 \\
Tutorización & & \\
\hline
\end{tabular}

\section{Discusión}

Las principales conclusiones parten de la autopercepción del profesorado respecto a su capacitación para enseñar el español como lengua vehicular, a pesar de la escasa formación específica recibida para ello en Andalucía objeto de este estudio. Esto viene a confirmar la necesidad de una formación específica no solo para este profesorado (ATAL), sino para todo el profesorado que se ocupa de la educación del alumnado inmigrante y también en la formación universitaria de los futuros docentes para Primaria y Secundaria. Es necesario aumentar la formación en la enseñanza del español como segunda lengua, conocer su especificidad, dominar las metodologías adecuadas, etcétera. 
Por lo tanto, se recomienda hacer una mejor planificación respecto a la integración social de este alumnado. Para ello será necesario que las aulas de apoyo lingüístico junto con las aulas ordinarias realicen un trabajo coordinado en esta línea. También es importante mejorar la integración del profesorado de las ATAL en el equipo docente de los centros y conseguir una mayor y mejor coordinación con el profesorado de las aulas de referencia.

\section{Referencias}

Agencia Andaluza de Evaluación Educativa (2015). Estudio sobre la organización y el funcionamiento de las Aulas Temporales de Adaptación Lingüística (ATAL). Sevilla: Consejería de Educación de la Junta de Andalucía. [Archived by WebCite® at http://www.webcitation.org/74jVDIQBp]

Arroyo, M. J. (2010). La lengua en la integración del alumnado inmigrante. Estudios de las aulas ALISO en la provincia de Segovia. Tesis Doctoral: Universidad de Valladolid, Segovia.

Arroyo, MJ. (2011). Las aulas y programas de inmersión lingüística para alumnado extranjero en España. Segundas Lenguas e Inmigración, 5, 114-139.

Barrios, ME. y Morales, L. (2012). Apoyo lingüístico inclusivo a alumnado no hispanohablante y aprendizaje de competencias curriculares en Educación Primaria. Porta Linguarum,17, 203221.

Consejería de Educación (2007). Orden de 15/01/2007, BOJA, 33. [Archived by WebCite ${ }^{\circledR}$ at http://www.webcitation.org/74cCkAEVw]
Consejo de Europa (2002). Marco común europeo de referencia para la Lenguas. [https://cvc.cervantes.es/ensenanza/biblioteca_ele/marco/cvc _mer.pdf]

Cruz del Pino, RM., Ruiz, C., García, S. y González, MC. (2011). Guía básica de educación intercultural. Sevilla: Consejería de Educación de la Junta de Andalucía.

Garcia, JA. y Moreno, I. (2003). La respuesta a las necesidades educativas de los hijos de inmigrantes en la Comunidad de Madrid. Madrid: Consejo Económico y Social de Madrid.

García, JA., Moreno, I. y Sánchez, P. (2008). Las aulas de enlace madrileñas, ¿espacios para el aprendizaje de la lengua vehicular o burbujas de acogida? Girona: Comunicación en el Sexto Simposio de Lengua, Cultura e Inmigración. [Archived by WebCite $®$ at http://www.webcitation.org/74jWDofj0] 